\title{
Consensus Protocols for Networks of Dynamic Agents
}

\author{
Reza Olfati Saber Richard M. Murray \\ Control and Dynamical Systems \\ California Institute of Technology \\ Pasadena, CA 91125 \\ e-mail: $\{$ olfati, murray\}@cds.caltech.edu
}

\begin{abstract}
In this paper, we introduce linear and nonlinear consensus protocols for networks of dynamic agents that allow the agents to agree in a distributed and cooperative fashion. We consider the cases of networks with communication time-delays and channels that have filtering effects. We find a tight upper bound on the maximum fixed time-delay that can be tolerated in the network. It turns out that the connectivity of the network is the key in reaching a consensus. The case of agreement with bounded inputs is considered by analyzing the convergence of a class of nonlinear protocols. A Lyapunov function is introduced that quantifies the total disagreement among the nodes of a network. Simulation results are provided for agreement in networks with communication time-delays and constrained inputs.
\end{abstract}

\section{Introduction}

An important problem that appears frequently in the context of coordination of multi-vehicle/multi-agent systems is the group agreement or consensus problem. Multi-agent systems have appeared broadly in several applications including formation flight of unmanned air vehicles (UAVs), clusters of satellites, self-organization, automated highway systems, and congestion control in communication networks.

The aforementioned applications justify the importance of design and analysis of consensus protocols to address agreement problems among communicating dynamic agents in a network. Such dynamic agents might or might not represent physical systems (or vehicles). In case where these dynamic agents are physical models, the input constraints for such systems have to be taken into account. This naturally leads us to the design and analysis of nonlinear consensus protocols.

Consensus problems make sense in the context of distributed systems [4] and have a long history in the field of Computer Science. In [1], the importance of the relation between graph Laplacian, a well-known matrix 0-7803-7896-2/03/\$17.00 C2003 IEEE in algebraic graph theory [2], and special cases of consensus problems were realized. In that work, all the vehicles have linear dynamics and the network has ideal links (i.e. the transfer function of the links is 1). In addition, certain Nyquist plots were useful in stability analysis for a multi-vehicle formation stabilization in Fax et al. . A more appropriate alternative to formation stabilization is to represent formations as rigid and unfoldable graphs/structures [7].

In this work, we do make use of a standard multivariable frequency domain analysis for convergence of linear consensus protocols. Two main contributions of this paper are to consider networks with time-delay and dynamic systems with bounded inputs. In [5], graph Laplacians appear in the context of attitude alignment of multiple spacecraft (i.e. a special agreement problem). An informal algorithm on attitude alignment for flocking of flying dynamic agents in a 3-D space was first introduced by Reynolds in 1987 without a convergence proof [8]. In [3], the authors made an attempt to prove the convergence of a modified version of the Reynolds attitude alignment algorithm for integratortype dynamic agents in $\mathbb{R}^{2}$. The key assumption in their proof is that in average with a high probability the graph remains connected in time.

The outline of the paper is as follows. Some background on algebraic graph theory is given in Section 2 . Linear protocols are presented in Section 3. The analysis for the case of networks with non-ideal links is discussed in Section 4. Nonlinear protocols are presented in Section 5. The simulation results are presented in Section 6. Finally, concluding remarks are made in Section 7.

\section{Preliminaries: Algebraic Graph Theory}

Let $\mathcal{G}=(\mathcal{V}, \mathcal{E})$ denote a graph with the set of vertices $\mathcal{V}$ and the set of edges $\mathcal{E}$. Each node is labeled by $v_{i} \in \mathcal{V}$, or $i \in \mathcal{I}:=\{1, \ldots, n\}$. Each edge is denoted by $e=\left(v_{i}, v_{j}\right)$ or $e=i j$ We refer to $v_{i}$ and $v_{j}$ as the tail and head of the edge $\left(v_{i}, v_{j}\right)$, respectively. We assume all the graphs in this paper are undirected. The
Proceedings of the American Control Conference
Denver, Colorado June 4-6, 2003 
orientation of the graph is a choice of heads and tails for each undirected edge. The set of edges of a fix orientation of the graph is denoted by $\mathcal{E}_{o}$. Thus, $\mathcal{E}_{o}$ contains one and only one of the two edges $i j, j i \in \mathcal{E}$. Let $n=|\mathcal{V}|$ and $m=\left|\mathcal{E}_{o}\right|$. The set of neighbors of node $i$ is denoted by $N_{i}=\{j: i j \in \mathcal{E}\}$. The degree of node $v_{i}$ is the number of its neighbors $\left|N_{i}\right|$ and is denoted by $\operatorname{deg}\left(v_{i}\right)$. The degree matrix is an $n \times n$ matrix defined as $\Delta=\Delta(\mathcal{G})=\left\{\Delta_{i j}\right\}$ where

$$
\Delta_{i j}:= \begin{cases}\operatorname{deg}\left(v_{i}\right) & , i=j \\ 0 & , i \neq j\end{cases}
$$

Let $A$ denote the adjacency matrix of $\mathcal{G}$. The the Laplacian of graph $\mathcal{G}$ is defined by

$$
L=\Delta-A
$$

An important feature of $L$ is that all the row sums of $L$ are zero and thus $e_{0}=(1,1, \ldots, 1)^{T} \in \mathbb{R}^{n}$ is an eigenvector of $L$ associated with the eigenvalue $\lambda=0$. There is an alternate way of defining the Laplacian matrix which is very beneficial for us. Fix an orientation of the graph and let $\mathcal{E}_{o}=\left\{e_{1}, e_{2}, \ldots, e_{m}\right\}$. Define the incidence matrix which is an $n \times m$ matrix as $C=\left[c_{i j}\right]$ where

$$
c_{i k}:= \begin{cases}+1 & \text { if } v_{i} \text { is the head of the edge } e_{k}, \\ -1 & \text { if } v_{i} \text { is the tail of the edge } e_{k}, \\ 0 & , \text { otherwise. }\end{cases}
$$

The Laplacian matrix satisfies the property $L=C C^{T}$. It is a well-known fact that this property holds regardless of the choice of the orientation of $\mathcal{G}$ [2]. Let $x_{i}$ denote a scalar real value assigned to $v_{i}$. Then, $x=\left(x_{1}, \ldots, x_{n}\right)^{T}$ denotes the state of the graph $\mathcal{G}$. We define the Laplacian potential of the graph as follows

$$
\Psi_{\mathcal{G}}(x)=\frac{1}{2} x^{T} L x
$$

From the new definition of the graph Laplacian, the following property of the Laplacian potential of the graph follows:

Lemma 1. (Laplacian potential) The Laplacian potential of a graph is positive semi-definite and satisfies the following identity:

$$
x^{T} L x=\sum_{i j \in \mathcal{E}_{o}}\left(x_{i}-x_{j}\right)^{2}
$$

Moreover, given a connected graph, $\Psi_{\mathcal{G}}(x)=0$ if and only if $x_{i}=x_{j}, \forall i, j$.

Proof. The positive semi-definiteness is due to $x^{T} L x=x^{T} C C^{T} x=\left\|C^{T} x\right\|^{2}$. In addition, if $\sum_{i j \in \mathcal{E}_{o}}\left(x_{i}-x_{j}\right)^{2}=0$, then for all edges $i j \in \mathcal{E}_{o}$, $x_{j}-x_{i}=0$. If the graph is connected, then the values of all nodes must be equal. The opposite statement is trivial, i.e. if the values of all nodes are equal, then $\Psi_{\mathcal{G}}(x)=0$.
Definition 1. (agreement) Let $x_{i}$ denote the value of node $v_{i}$ for all $i \in \mathcal{I}$. We say nodes $v_{i}$ and $v_{j}$ agree if and only if $x_{i}=x_{j}$. Similarly, we say they disagree if and only if $x_{i} \neq x_{j}$.

According to Lemma 1, the Laplacian potential of the graph $\mathcal{G}$ is a measure of total disagreement among all nodes. If at least two neighboring nodes of $\mathcal{G}$ disagree, then $\Psi_{\mathcal{G}}(x)>0$. As a result, minimizing $\Psi_{\mathcal{G}}(x)$ is equivalent to reaching a consensus. We formalize this idea in the rest of the paper.

Definition 2. (consensus) Let the value of all nodes $x$ be the solution of the following differential equation:

$$
\dot{x}=f(x), \quad x(0)=x^{0} \in \mathbb{R}^{n}
$$

In addition, let $\chi: \mathbb{R}^{n} \rightarrow \mathbb{R}$ be a multi-input singleoutput operation on $x=\left(x_{1}, \ldots, x_{n}\right)^{T}$ that generates a decision-value $y=\chi(x)$. We say all the nodes of the graph have reached consensus w.r.t. $\chi$ in finite time $T>0$ if and only if all the nodes agree and $x_{i}(T)=\chi(x(0)), \forall i \in I$. Similarly, let $x=x^{*}$ be a globally/locally asymptotically stable equilibrium of (6). We say all the nodes of the graph with initial values $x_{i}^{0}$ have globally/locally asymptotically reached consensus regarding $\chi$ if and only if $x_{i}^{*}=\chi(x(0)), \forall i \in \mathcal{I}$

Example 1. Some of the common examples of the operation $\chi$ are given in the following:

$$
\begin{aligned}
& \chi(x)=\operatorname{Ave}(x)=\frac{1}{n} \sum_{i=1}^{n} x_{i} \\
& \chi(x)=\operatorname{Max}(x)=\max \left\{x_{1}, x_{2}, \ldots, x_{n}\right\} \\
& \chi(x)=\operatorname{Min}(x)=\min \left\{x_{1}, x_{2}, \ldots, x_{n}\right\}
\end{aligned}
$$

The corresponding consensus of these operations are referred to as the average-consensus, the max-consensus, and the min-consensus, respectively. This suggests a general name of $\chi$-consensus for an agreement problem regarding the operation $\chi$.

According to Lemma 1, the Laplacian potential of the graph $\mathcal{G}$ is a measure of total disagreement among all nodes. If at least two neighboring nodes of $\mathcal{G}$ disagree, then $\Psi_{\mathcal{G}}(x)>0$. As a result, minimizing $\Psi_{\mathcal{G}}(x)$ is equivalent to reaching a consensus. This the key in the design of a consensus protocol.

Lemma 2. (connectivity and graph Laplacian [2]) Assume graph $\mathcal{G}$ has $\mathrm{c}$ connected components, then

$$
\operatorname{rank}(L)=\operatorname{rank}(C)=n-c
$$

Particularly, for a connected graph with $c=1$, $\operatorname{rank}(L)=n-1$.

Based on Lemma 2, the algebraic multiplicity of the zero eigenvalue of $L$ is 1 , if and only if the graph is connected. In addition, for an undirected graph, all the other eigenvalues of $\mathcal{G}$ are positive and real. 


\section{Linear Consensus Protocols}

In this section, we present two linear consensus protocols for networks of integrators both with and without communication time-delays.

Theorem 1. Let $\mathcal{G}$ be a connected graph and suppose each node of $\mathcal{G}$ applies the following distributed linear protocol

$$
u_{i}(t)=\sum_{j \in N_{i}}\left(x_{j}(t)-x_{i}(t)\right)
$$

Then, the vector of the value of the nodes $x$ is the solution of a gradient system associated with the Laplacian potential $\Psi_{G}(x)$, i.e.

$$
\dot{x}=-L x=-\nabla \Psi_{G}(x), \quad x(0) \in \mathbb{R}^{n}
$$

In addition, all the nodes of the graph globally asymptotically reach an average-consensus, i.e. let $x^{*}=$ $\lim _{t \rightarrow+\infty} x(t)$, then $x_{i}^{*}=x_{j}^{*}=\operatorname{Ave}(x(0)), \forall i, j, i \neq j$.

Proof. Let $x^{*}$ be an equilibrium of the system $\dot{x}=$ $-L x$. Then $L x^{*}=0$ and thus $x^{*}$ is the eigenvector of the Laplacian $L$ associated with the zero eigenvalue $\lambda_{1}=0$. On the other hand, we have

$$
\Psi_{\mathcal{G}}\left(x^{*}\right)=\frac{1}{2}\left(x^{*}\right)^{T} L x^{*}=0
$$

and from connectivity of $\mathcal{G}$ it follows that $x_{i}^{*}=x_{j}^{*}=$ $a, \forall i, j$, i.e. $x^{*}=(a, \ldots, a)^{T}, a \in \mathbb{R}$. Notice that $\sum_{i=1}^{n} u_{i}=0$. Thus, $\bar{x}=A v e(x)$ is an invariant quantity, i.e. $\dot{\bar{x}}=0$. This implies $\operatorname{Ave}\left(x^{*}\right)=\operatorname{Ave}(x(0))$. But $\operatorname{Ave}\left(x^{*}\right)=a$, therefore $x_{i}^{*}=\operatorname{Ave}(x(0)$ for all the nodes $i \in \mathcal{I}$. Observe that all the eigenvalues of $-L$ are negative, except for the single zero eigenvalue. Thus, any solution of the system asymptotically converges to a point $x^{*}$ in the eigenspace associated with $\lambda_{1}=0$. This implies that an average-consensus is globally asymptotically reached by all the nodes.

Remark 1. It turns out that the planar version of Reynolds algorithm is the same as the linear protocol in (9). This relies on two assumptions: i) the edges of the graph are determined according to the closest spatial neighbors of each agent, and ii) the graph Laplacian remains invariant during flocking.

An elementary method to analyze the convergence of the protocol given in Theorem 1 is to use the Laplace transform of $\dot{x}=-L x$ as follows. We have $X(s)=$ $G_{0}(s) x(0)$ where $G_{0}(s)$ is a multi-input multi-output (MIMO) transfer function given by

$$
G_{0}(s)=\left(s I_{n}+L\right)^{-1} \text {. }
$$

Here, the subscript 0 in $G_{0}(s)$ implies zero communication time-delay.
Note. Throughout the paper, we use usual notation in the Laplace (or frequency) domain, i.e. $X(s)$ and $X(j \omega)$ are the Laplace transform and the Fourier transform of the signal $x(t)$, respectively.

A sufficient condition that the aforementioned protocol converges is that all the poles of $G(s)$ have to be on the LHP except for an isolated pole at zero.

The following result gives a non-conservative bound on the communication delay between two nodes of the network such that still an average-consensus can be reached.

Theorem 2. Suppose that each node $v_{i}$ of a connected graph $\mathcal{G}$ receives the information (i.e. $x_{j}$ ) from its neighboring nodes after a fixed delay $\tau>0$ and applies the following linear protocol

$$
u_{i}(t)=\sum_{j \in N_{i}}\left(x_{j}(t-\tau)-x_{i}(t-\tau)\right)
$$

Then, the the value of the nodes is the solution of the following linear delay differential equation:

$$
\dot{x}=-L x(t-\tau), \quad x(0) \in \mathbb{R}^{n}
$$

In addition, all the nodes of the graph globally asymptotically reach an average-consensus if and only if either of the following two equivalent conditions are satisfied:

i) $\tau \in\left(0, \tau^{*}\right)$ with $\tau^{*}=\frac{\pi}{2 \lambda_{n}}, \lambda_{n}=\lambda_{\max }(L)$.

ii) The Nyquist plot of $\Gamma(s)=e^{-\tau s} / s$ has a zero encirclement around $-1 / \lambda_{k}, \forall k>1$.

Moreover, for $\tau=\tau^{*}$ the system has a globally asymptotically stable oscillatory solution with the frequency $\omega=\lambda_{n}$.

Proof. See the proof of Theorem 2 in [6].

\section{Linear Protocols for Networks with Non-ideal Links}

In general, the characteristic of each link can be represented by a stable transfer function $h(s)$. This can represent a time-delay, a Pade approximation of a timedelay (an all-pass filter), or possibly the distortion or filtering effects in a communication link. Assume all the links have the same characteristic $h(s)$ and let $\hat{X}_{j}(s)$ denote the filtered output of the link with an input $X_{j}(s)$, i.e. $\hat{x}_{j}(t):=h(t) * x_{j}(t)$. Then, the consensus protocol for this network with non-ideal links $(h(s) \neq 1)$ can be expressed as

$$
u_{i}(t)=\sum_{j \in N_{i}}\left(\hat{x}_{j}(t)-\hat{x}_{i}(t)\right), \quad \forall i \in \mathcal{I}
$$


where is the special case of a time-delay with $h(s)=$ $e^{-\tau s}$ reduces to (12). Clearly, $\sum_{i=1}^{n} u_{i}(t)=0, \forall t \geq 0$ and $\bar{x}=\operatorname{Ave}(x)$ is an invariant quantity. Thus, if the protocol in (14) converges, the decision-value for all the agents is $A v e(x(0))$ regardless of the choice of $H(s)$. After performing a frequency domain analysis similar to the one presented in the proof of Theorem 2, one obtains $X(s)=G(s) x(0)$ with $G(s)=\left(s I_{n}+h(s) L\right)^{-1}$ and $Z(s):=s I_{n}+h(s) L$ that leads to the following Nyquist convergence criterion for the protocol in (14):

$$
\frac{1}{\lambda_{k}}+\Gamma(s)=0, \quad k>1
$$

with $\Gamma(s)=h(s) / s$. In other words, if the net encirclement of the Nyquist plot of $\Gamma(s)$ around $-1 / \lambda_{k}, k>$ 1 is zero, then (14) globally asymptotically converges. This result has a striking similarity to a result in [1], despite the fact that the agents have a different dynamics than the ones used in [1]. The nature of this similarity is due to the fact that analyzing the stability of any linear system with delay can be addressed using Laplace transforms and will reduce to a frequency domain analysis.

In the most general form, let $h_{i j}(s)=h_{j i}(s)$ denote the characteristic of the link that connects node- $i$ and node- $j$. Define the adjacency matrix in the Laplace domain, $A(s)$ as a matrix with elements $h_{i j}(s)$ when there is a an edge $i j$ and zero where there are no edges. The degree matrix in the Laplace domain is a diagonal matrix $\Delta(s)$ with the $i$ th element given by

$$
\Delta_{i i}(s):=\sum_{j \in N_{i}} h_{i j}(s)
$$

We define the Laplacian of the network in the Laplace domain as $L(s)=\Delta(s)-A(s)$. Then, $G(s)=\left(s I_{n}+\right.$ $L(s))^{-1}$ is the main MIMO transfer function of interest satisfying $X(s)=G(s) x(0)$. In general, if all the $h_{i j}(s)$, the stability of $G(s)=\left(s I_{n}+L(s)\right)^{-1}$ can be directly checked by finding all of its poles. In the special case with $h_{i j}(s)=h(s)$ for $h_{i j}(s) \not \equiv 0$, we obtain $L(s)=h(s) L$ and thus $G(s)=\left(s I_{n}+h(s) L\right)^{-1}$. The benefit of defining a general Laplacian matrix $L(s)$ in the Laplace domain is that one can analyze linear protocols for more realistic communication networks where the time-delay of all the links are not necessarily equal.

\section{Action Graphs and Nonlinear Protocols}

The problem of attitude alignment for robots and spacecraft is a special case of the consensus problem. For these physical systems, it is not reasonable to assume that their attitude can change by an unbounded value, i.e. the input torque is bounded. This suggests development of consensus protocols that guarantee the overall input of each node remains bounded. This naturally leads to design and analysis of nonlinear consensus protocols. First, we introduce the notion of action graphs as a general design tool for nonlinear consensus protocols.

Let $(\mathcal{V}, E)$ be a graph with the set of vertices $\mathcal{V}$ and the set of edges $\mathcal{E}$. An action graph, denoted by $\mathcal{G}=(\mathcal{V}, \mathcal{E}, \Phi)$, is a graph $(\mathcal{V}, \mathcal{E})$ augmented with a set of action functions $\Phi$ with elements $\phi_{i j}: \mathbb{R} \rightarrow \mathbb{R}$ that are associated with the edges $e_{i j}=\left(v_{i}, v_{j}\right) \in \mathcal{E}$ of the graph. Throughout this paper, we assume that an action function $\phi(x)$ satisfies the following properties:

i) $\phi(x)$ is continuous and locally Lipschitz,

ii) $\phi(x)=0 \Leftrightarrow x=0$,

iii) $\phi(-x)=-\phi(x), \forall x \in \mathbb{R}$,

iv) $(x-y)(\phi(x)-\phi(y))>0, \forall x \neq y$.

Let $\psi_{i j}(x)=\int_{0}^{x} \phi_{i j}(s) d s$, then $\psi_{i j}(x)$ associated with $e_{i j} \in \mathcal{E}$ is called the edge cost. Define the set of edge costs as $\Psi=\left\{\psi_{i j}:\left(v_{i}, v_{j}\right) \in \mathcal{E}\right\}$. Then $\mathcal{G}=(\mathcal{V}, \mathcal{E}, \Psi)$ is called a cost graph.

Remark 2. Clearly, corresponding to any action graph with measurable action functions, there exists a cost graph. The opposite holds provided that each cost in is a continuously differential convex function with a global minimum at $x=0$.

In the special case where all the edge action functions are equal to $\phi$, we use the notation $\mathcal{G}=(\mathcal{V}, \mathcal{E}, \phi)$ to represent the action graph and call it a uniaction graph. The main focus of this paper is the application of uniaction graphs in providing consensus protocols and algorithms for a network of dynamic systems.

Consider a dynamic graph in which each node is a dynamic system

$$
\dot{x}_{i}=f\left(x_{i}, u_{i}\right), \quad i \in \mathcal{I}
$$

For now, we assume each node is an integrator, i.e. $\dot{x}_{i}=u_{i}, \forall i \in \mathcal{I}$.

Theorem 3. (Consensus with Nonlinear Protocol) Consider a dynamic action graph $\mathcal{G}=(\mathcal{V}, \mathcal{E}, \Phi)$ with integrator nodes and suppose that $(\mathcal{V}, \mathcal{E})$ is a connected graph. Assume that all the edge actions are symmetric $\left(\phi_{i j}=\phi_{j i}\right)$ and each node applies the following input

$$
u_{i}=\sum_{j \in N_{i}} \phi_{i j}\left(x_{j}-x_{i}\right), \forall i \in \mathcal{I}
$$

where $N_{i}=\{j \in \mathcal{I}: i j \in \mathcal{E}\}$. Then, there exists a common decision-value $x^{*}=A v e(x(0))$ given by that is reached globally asymptotically by every node in the action graph. 
Proof. Let $\alpha=\operatorname{Ave}(x)$ and notice that due to $\sum_{i=1}^{n} u_{i}=0, \alpha$ is an invariant quantity, i.e. $\dot{\alpha}=0$ and $\alpha(t)=\operatorname{Ave}(x(0))$, for all $t \geq 0$. We can write $x$ as

$$
x=\alpha \mathbf{1}+\delta
$$

where $\mathbf{1}=(1, \ldots, 1)^{T}$ and $\delta$ is called the disagreement vector. Notice that $\sum_{i} \delta_{i}=0$ and $\dot{x}=\dot{\delta}$, thus the disagreement vector satisfies the following disagreement dynamics

$$
\dot{\delta}_{i}=\sum_{j \in N_{i}} \phi_{i j}\left(\delta_{j}-\delta_{i}\right), \quad i=1, \ldots, n
$$

due to $x_{j}-x_{i}=\delta_{j}-\delta_{i}$, for all $i, j$. Defining the group disagreement function as

$$
V(\delta)=\|\delta\|^{2}
$$

we get

$$
\begin{aligned}
\dot{V}(\delta) & =2 \sum_{i=1} \sum_{j \in N_{i}} \delta_{i} \phi_{i j}\left(\delta_{j}-\delta_{i}\right) \\
& =\sum_{(i, j) \in \mathcal{E}} \delta_{i} \phi_{i j}\left(\delta_{j}-\delta_{i}\right)+\delta_{j} \phi_{j i}\left(\delta_{i}-\delta_{j}\right) \\
& =-\sum_{(i, j)}\left(\delta_{j}-\delta_{i}\right) \phi_{i j}\left(\delta_{j}-\delta_{i}\right) \leq 0 .
\end{aligned}
$$

Notice that $\dot{V}(\delta)=0$ implies $\delta_{i}=\delta_{j}$ for all the edges $(i, j) \in \mathcal{E}$. Since the graph is connected, we have $\delta_{i}=\delta_{j}$ for all $i, j \in \mathcal{I}$. By definition of $\delta, \sum_{i} \delta_{i}=0$ thus $\delta_{i}=0$ for all $i$. In other words, $\delta \neq 0$ implies $\dot{V}(\delta)<0$ and therefore the group disagreement function $V(\delta)$ is a valid Lyapunov function for the disagreement dynamics. As a result, $\delta=0$ is globally asymptotically stable for $(20)$, i.e. $x(t) \rightarrow \alpha 1$ as $t \rightarrow+\infty$ and averageconsensus is globally asymptotically achieved.

Remark 3. Due to symmetry of action functions and property iii), the following identity holds

$$
\phi_{j i}\left(\delta_{i}-\delta_{j}\right)=-\phi_{i j}\left(\delta_{j}-\delta_{i}\right)
$$

In lack of the symmetry of action functions, one can define the following set of symmetric action functions

$$
\phi_{i j}^{s}(z)=\frac{\phi_{i j}(z)+\phi_{j i}(z)}{2}
$$

and replace the action functions in protocol (A1) with their symmetric counterparts and still the same result holds.

\section{Simulation Results}

We consider solving average-consensus problem for graphs $\mathcal{G}_{a}$ and $\mathcal{G}_{b}$ shown in Figure 1 . Figures 2 and 3 show the simulation results for the nonlinear consensus protocol for a network with information flow $\mathcal{G}_{b}$. Apparently, agreement is achieved with bounded inputs. Figure 4 shows the state trajectories of $n=10$ nodes for a network with communication time-delay $\tau$

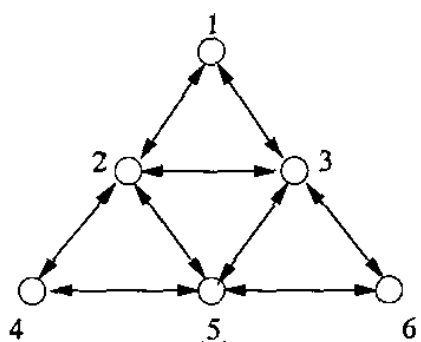

(a)

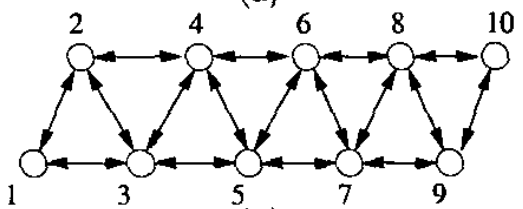

(b)

Figure 1: Undirected graphs used for consensus problems: a) $\mathcal{G}_{a}$ and b) $\mathcal{G}_{b}$.

for $\tau=0,0.5 \tau_{\max }, \tau_{\max }=\pi / 2 \lambda_{\max }\left(\mathcal{G}_{a}\right)=0.266$ for a zero-mean random set of initial conditions. Clearly, the agreement is achieved for the cases with $\tau<\tau_{\max }$ in Figures 4(a) and (b). For the case with $\tau=\tau_{\max }$, synchronous oscillations are demonstrated in Figure 4(c). $A$ third-order Pade approximation is used to model the time-delay as a finite-order LTI system.

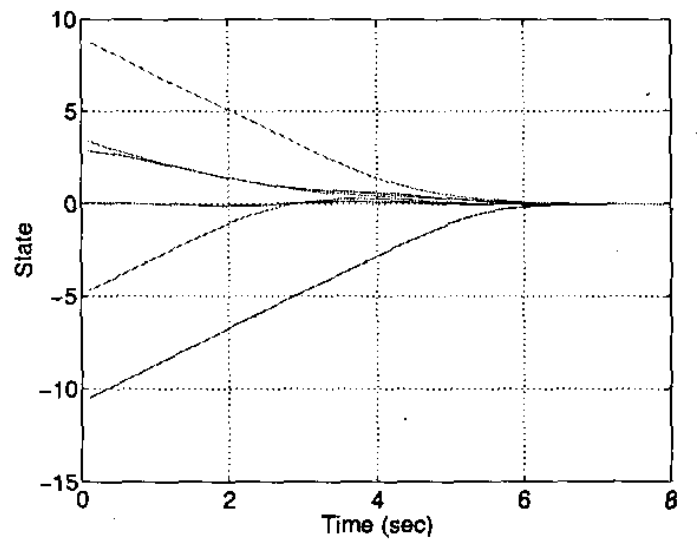

Figure 2: State trajectories for agreement with nonlinear protocol on $\mathcal{G}_{o}$

\section{Conclusions}

In this paper, we introduced linear and nonlinear consensus protocols for a network of dynamic agents with undirected information flow that solves an averageconsensus problem in a distributed way. We discussed how the convergence analysis is done for the cases where the characteristic function of the communication links are not equal to 1 . This includes links with time-delay and possibly distortion and filtering effects. We used standard tools from multivariable control and linear control theory such as Nyquist plots to analyze the convergence properties of the linear protocols. For Proceedings of the American Control Conference 


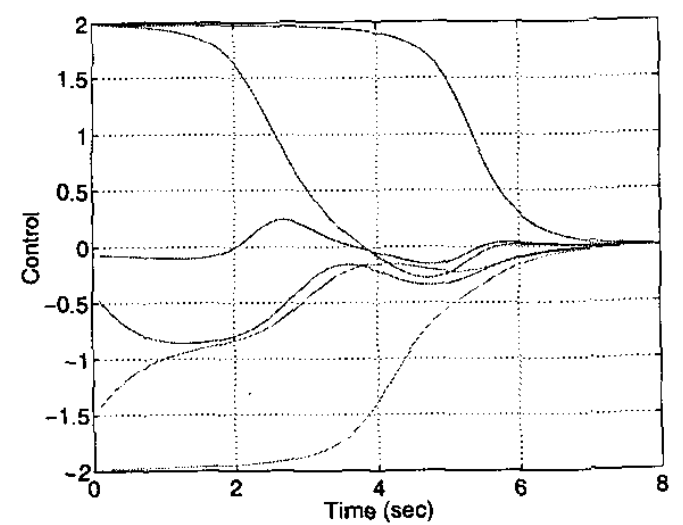

Figure 3: Bounded input for agreement with nonlinear protocol on $\mathcal{G}_{a}$

the analysis of the nonlinear protocols, we introduced the notion of action graphs and constructed disagreement costs that are minimized by nonlinear consensus protocols in a distributed way. Simulation results were presented that are consistent with our theoretical results.

\section{References}

[1] A. Fax and R. M. Murray. Information Flow and Cooperative Control of Vehicle Formations. The 15th IFAC World Congress, June 2002.

[2] C. Godsil and G. Royle. Algebraic Graph Theory, volume 207 of Graduate Texts in Mathematics. Springer, 2001.

[3] A. Jadbabaie, J. Lin, and S. A. Morse. Coordination of groups of mobile agents using nearest neighbor rules. To appear in the IEEE Trans, on Automatic Control, 2002.

[4] N. A. Lynch. Distributed Algorithms. Morgan Kaufmann Publishers, Inc., 1997.

[5] M. Mesbahi. On a dynamic extension of the theory of graphs. Proc. of the American Control Conference, Anchorange, AL, May 2002.

[6] R. Olfati Saber and Murray R. M. Consensus protocols for undirected networks of dynamic agents with communication time-delays. Technical Report CIT-CDS 03-007, California Institute of Technology, Control and Dynamical Systems, Pasadena, California, March 2003.

[7] R. Olfati-Saber and R. M. Murray. Graph Rigidity and Distributed Formation Stabilization of MultiVehicle Systems. Proceedings of the IEEE Int. Conference on Decision and Control, Dec. 2002.

[8] C. W. Reynolds. Flocks, herds, and schools: a distributed behavioral model. Computer Graphics (ACM SIGGRAPH '87 Conference Proceedings), 21(4):25-34, July 1987.

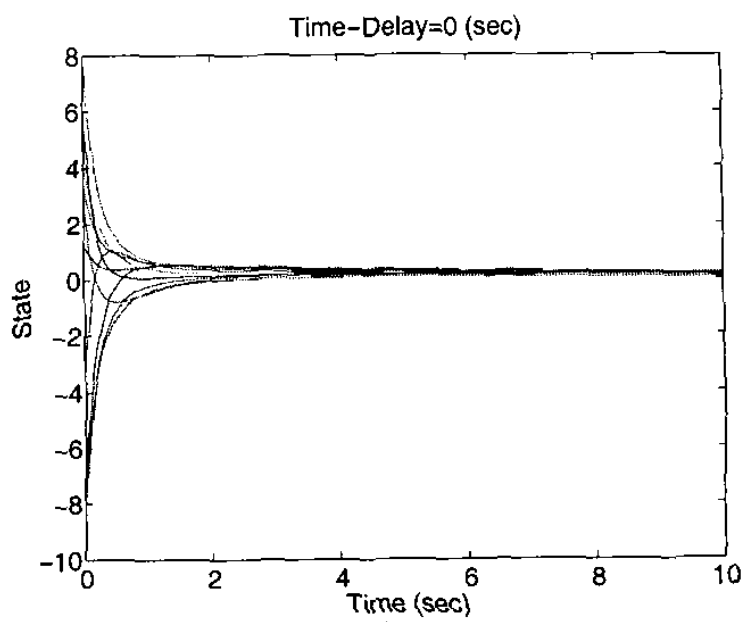

(a)

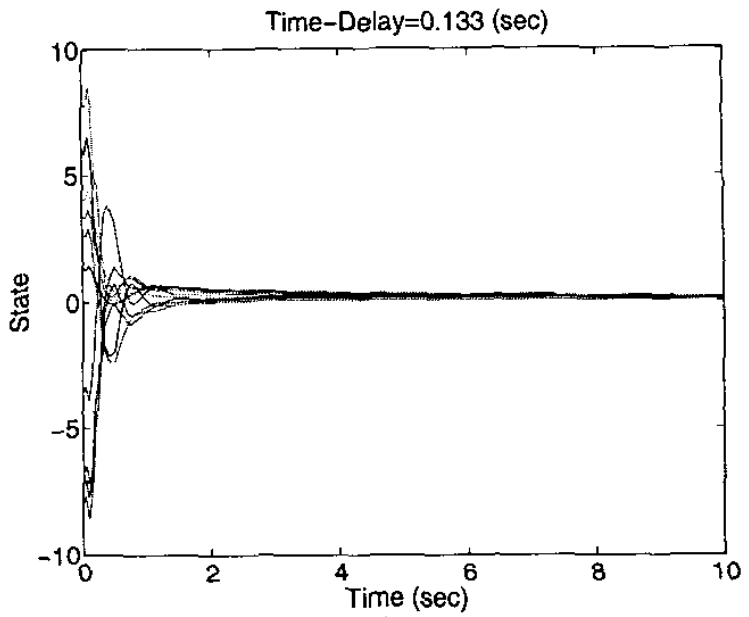

(b)

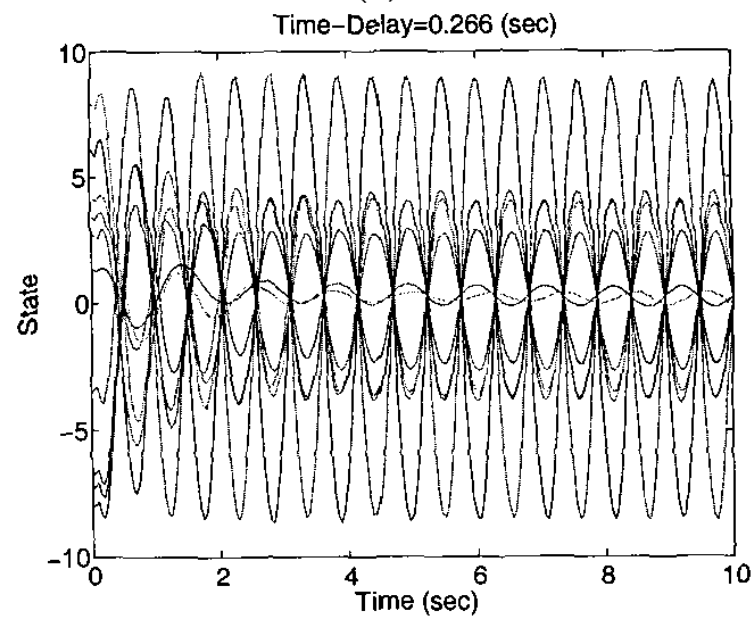

(c)

Figure 4: Consensus problem with communication timedelay on $\mathcal{G}_{b}$ in Figure 1: (a) $\tau=0$, (b) $\tau=$ $0.5 \tau_{\max }$, and (c) $\tau=\tau_{\max }$. 